Center for Mathematical Studies in Economics and Management Science Northwestern University, Evanston: I11inois 60208

Discussion Paper No. 1133

DUAL REDUCTION AND ELEMENTARY GAMES

by

Roger B. Myerson*

June 1995

Abstract: Consider the linear incentive constraints that define the correlated equilibria of a game. The duals of these constraints generate Markov chains on the players' strategy sets. The stationary distributions for these Markov chains can be interpreted as the strategies in a reduced game, which is called a dual reduction. Any equilibrium of a dual reduction is an equilibrium of the original game. We say that a game is elementary if all incentive constraints can be satisfied as strict inequalities in a correlated equilibrium. Any game can be reduced to an elementary game by iterative dual reduction.

Acknowledgements. Support from the National Science Foundation grant SES-9308139 is gratefully acknowledged.

*Department of Managerial Economics and Decision Sciences, J. L. Kellogg Graduate School of Management, Northwestern University, Evanston, IL 60208 . (Internet: myerson@casbah.acns.nwu.edu) 


\section{DUAL REDUCTION AND ELEMENTARY GAMES}

by Roger B. Myerson

\section{Introduction}

Robert Aumann (1974) defined correlated equilibria to include all jointly randomized strategies that are feasible for the players in a game when they can communicate with each other and with a mediator, assuming that each player will rationally choose his (or her) own strategy after all communication is finished. The set of correlated equilibria thus contains the set of Nash equilibria as a subset. However, the set of all correlated equilibria is generally much easier to characterize mathematically than the smaller set of Nash equilibria. That is, allowing the possibility of mediated communication greatly simplifies equilibrium analysis of general games.

This paper develops some general mathematical methods for simplifying and analyzing strategic-form games with communication, when correlated equilibrium is taken as the basic solution concept. We denote a game in strategic form by

$$
\Gamma=\left(\mathrm{N},\left(\mathrm{C}_{\mathrm{i}}\right)_{i \in \mathrm{N}}, \quad\left(\mathrm{U}_{\mathrm{i}}\right)_{\mathrm{i} \in \mathrm{N}}\right),
$$

where $\mathrm{N}$ denotes the set of players, $C_{i}$ denotes the set of pure strategies of player $i$, and $U_{i}: x_{j \in N} C_{j} \rightarrow \mathbf{R}$ denotes the utility function of player $i$. Throughout this paper, we assume that the set of players $\mathrm{N}$ and the pure strategy sets $C_{i}$ are nonempty finite sets.

Given such a game $\Gamma$, the set of (pure) strategy profiles is denoted by

$$
C=x_{j \in N} C_{j} .
$$

For each player $i$, the set of strategy profiles for the players other than $i$ is

$$
\mathrm{C}_{-i}=\times_{j \in \mathrm{N}-\mathrm{i}} \mathrm{C}_{j} .
$$

A strategy profile in $C$ may be denoted $c=\left(c_{i}\right)_{i \in N}$. We may write $\left(c_{-i}, d_{i}\right)$ 
to denote the strategy profile that differs from $c$ only in that $d_{i}$ is i-component.

For any finite set $S$, we let $\Delta(S)$ denote the set of probability

distributions over the set $\mathrm{S}$. So a correlated strategy for the players in $\mathrm{N}$ is any probability distribution in $\Delta(C)$. That is, $\mu$ is a correlated strategy iff $\mu=(\mu(\mathrm{c}))_{\mathrm{c} \in \mathrm{C}}$ satisfies

$$
\begin{aligned}
& \mu(\mathrm{c}) \geq 0, \quad \forall \mathrm{c} \in \mathrm{C}, \\
& \Sigma_{\mathrm{c} \in \mathrm{C}} \mu(\mathrm{c})=1 .
\end{aligned}
$$

We may think of any such correlated strategy $\mu$ as being implemented with the help of a mediator. First the mediator selects a recommended strategy profile according to the probability distribution $\mu$, selecting any strategy profile $c$ with probability $\mu(\mathrm{c})$. Then the mediator confidentially tells each player $i$ the component $c_{i}$ of this strategy profile that is recommended for him. But after the mediator communicates these recommendations, the actual strategy choice in $C_{i}$ is up to player $i$, who is free to obey or disobey the mediator's recommendations. The mediator's correlated strategy is a correlated equilibrium iff it would be a Nash equilibrium for all players to obey the mediator's recommendations. That is, a correlated equilibrium is any correlated strategy $\mu$ such that

$$
\Sigma_{c_{-i} \in C_{-i}} \mu(c)\left(U_{i}\left(c_{-i}, d_{i}\right)-U_{i}(c)\right) \leq 0, \quad \forall i \in N, \quad \forall c_{i} \in C_{i}, \quad \forall d_{i} \in C_{i} .
$$

The inequalities in (1) are incentive constraints, and they stipulate that each player i could not expect to increase his expected utility by using strategy $d_{i}$ when he is told by the mediator to use the strategy $c_{i}$, assuming that the mediator's recommendations are generated according to the distribution $\mu$, and assuming that all other players are expected to obey the mediator's 
recommendations.

The weak inequalities in the incentive constraints (1) allow that a player might be indifferent between obeying and disobeying the mediator's recommendation in a correlated equilibrium. If a player would be indifferent between conforming to the correlated equilibrium and deviating from it, then we need to worry whether the slightest perturbation of incentives and beliefs might cause the equilibrium to unravel. For this reason, game theorists since Selten (1975) have studied equilibrium refinements such as perfect equilibrium, to identify when such players' indifference to deviation should be considered problematic in an equilibrium, and when it should not. (For a detailed review of this literature, see van Damme, 1991, for example.)

There are only a few papers which have tried to extend equilibrium refinements such as perfectness to games where correlated equilibrium is the basic solution concept. (See Myerson, 1986, and Dhillon and Mertens, 1992.) In this paper, we offer a new perspective on this question. We begin in Section 2 by defining a class of elementary games where the problem of indifference to deviation does not arise in correlated equilibria. Then we develop a new technique, called dual reduction, that will enable us to reduce any game in strategic form to such an elementary game. The mathematical foundations of dual reduction are taken from Nau and McCardle's (1990) proof of existence of correlated equilibria, which we review in Section 3 . The definition of dual reduction is developed in Section 4, and in Section 5 we show that iterative dual reduction can always generate an elementary game. Examples and refinements are discussed in Sections 6 and 7 , and Section 8 contains the longer proofs. 


\section{Elementary games}

Given a strategic-form game $\Gamma$ as above, we say that a correlated equilibrium $\mu$ has elementary incentives if $\mu$ satisfies all the incentive constraints (1) as strict inequalities; that is,

$$
\Sigma_{c_{-i} \in C_{-i}} \mu(c)\left(U_{i}\left(c_{-i}, d_{i}\right)-U_{i}(c)\right)<0, \quad \forall i \in N, \quad \forall c_{i} \in C_{i}, \forall d_{i} \in C_{i} .
$$

Notice that this definition implies that every pure strategy must have positive probability in $\mu$,

$$
\Sigma_{c_{-i} \in C_{-i}} \mu(c)>0, \quad \forall i \in N, \quad \forall c_{i} \in C_{i} .
$$

That is, a correlated equilibrium has elementary incentives if every pure strategy of every player has a positive probability of being recommended, and any player would strictly decrease his expected payoff by unilaterally deviating from any recommended strategy, when all other players are expected to obey the recommendations of the correlated equilibrium.

If $\mu$ is any correlated equilibrium and $\hat{\mu}$ is a correlated equilibrium with elementary incentives then, for any number $\varepsilon$ between 0 and $1,(1-\varepsilon) \mu+\varepsilon \hat{~ i s ~}$ also a correlated equilibrium with elementary incentives. This fact is easy to verify, using the linearity of the incentive constraints. Thus, if a game has any correlated equilibrium with elementary incentives, then every correlated equilibrium can be approximated arbitrarily closely by correlated equilibria with elementary incentives. That is, we have the following fact.

\section{Proposition 1 . If the set of correlated equilibria with elementary} incentives is nonempty, then it is dense in the set of all correlated equilibria. 
So let us say that the game $\Gamma$ is elementary if $\Gamma$ has correlated equilibria with elementary incentives. For such elementary games, any player can be motivated to choose any pure strategy, with no indifference problems. Any indifference that may arise in a correlated equilibrium can be eliminated by arbitrarily small perturbations within the correlated equilibrium set. So correlated-equilibrium refinements that generalize Selten's perfectness concept should be unnecessary for such elementary games.

This concept of elementary games is quite restrictive (and so it may be remarkable that we will be able to reduce all games to elementary games). To illustrate this concept, let us consider three well-known examples.

$\begin{array}{cccr} & & & \text { Player } 2 \\ & & \mathrm{x}_{2} & \mathrm{y}_{2} \\ \text { Player 1 } & \mathrm{x}_{1} & 3,2 & 0,0 \\ & \mathrm{y}_{1} & 0,0 & 2,3\end{array}$

Figure 1. The Battle of the Sexes Game

The Battle of Sexes game (Figure 1) is an elementary game. The correlated equilibrium $\mu$ such that $\mu\left(\mathrm{x}_{1}, \mathrm{x}_{2}\right)=.5=\mu\left(\mathrm{y}_{1}, \mathrm{y}_{2}\right)$ has elementary incentives, because either player i would expect to strictly decrease his payoff by unilaterally deviating to $y_{i}$ when $x_{i}$ is recommended, or by deviating to $x_{i}$ when $y_{i}$ is recommended. 


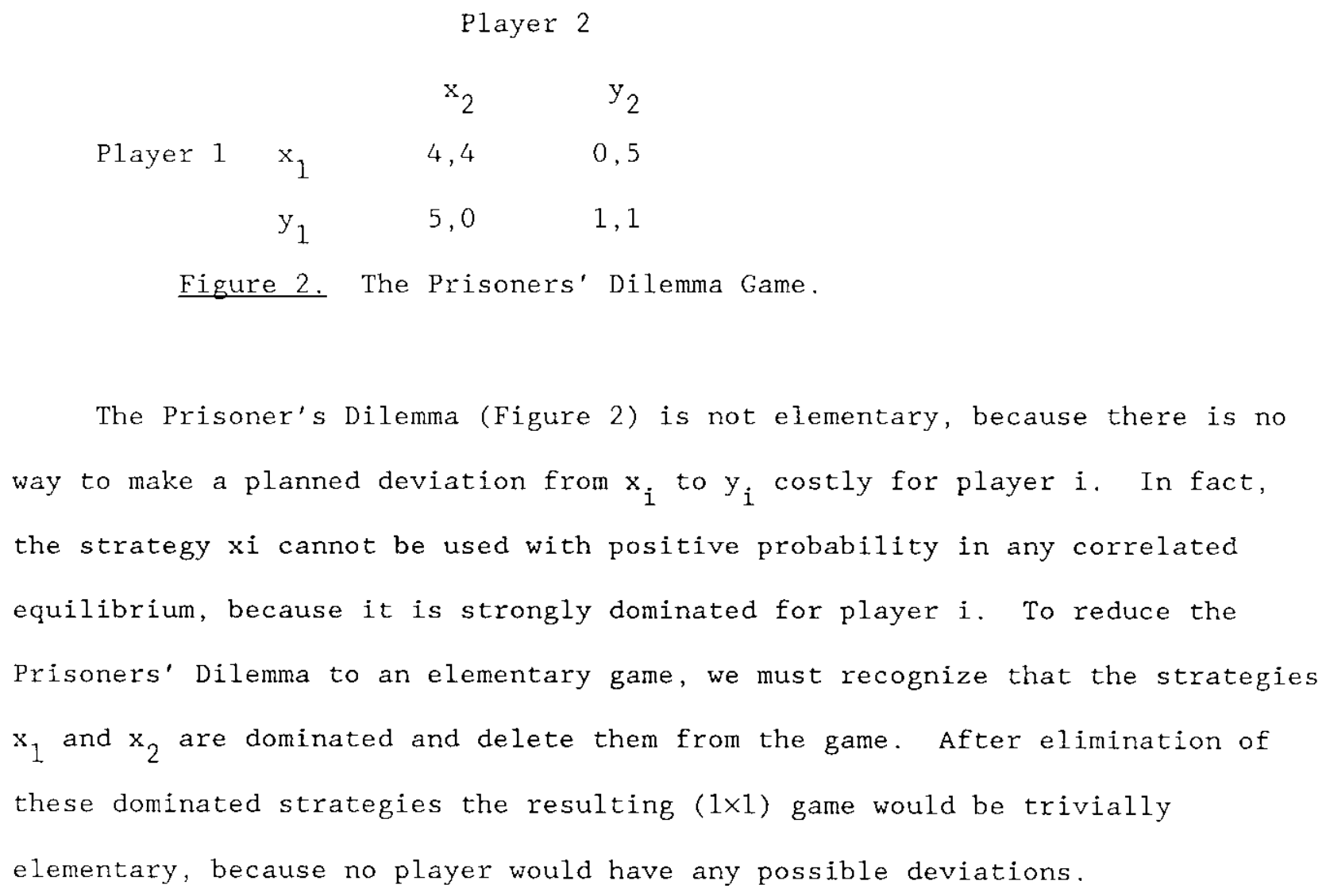

The Prisoner's Dilemma (Figure 2) is not elementary, because there is no way to make a planned deviation from $x_{i}$ to $y_{i}$ costly for player $i$. In fact, the strategy $x i$ cannot be used with positive probability in any correlated equilibrium, because it is strongly dominated for player $i$. To reduce the Prisoners' Dilemma to an elementary game, we must recognize that the strategies $\mathrm{x}_{1}$ and $\mathrm{x}_{2}$ are dominated and delete them from the game. After elimination of these dominated strategies the resulting ( $1 \times 1$ ) game would be trivially elementary, because no player would have any possible deviations.

Player 2

$\begin{array}{rrcc} & & \mathrm{x}_{2} & \mathrm{y}_{2} \\ \text { Player 1 } & \mathrm{x}_{1} & 1,-1 & -1,1 \\ & \mathrm{y}_{1} & -1,1 & 1,-1 \\ \text { Figure 3. The Matching Pennies Game }\end{array}$

The Matching Pennies game (Figure 3) is also not elementary. This game has only one correlated equilibrium, which is such that

$$
\mu\left(\mathrm{x}_{1}, \mathrm{x}_{2}\right)=\mu\left(\mathrm{x}_{1}, \mathrm{y}_{2}\right)=\mu\left(\mathrm{y}_{1}, \mathrm{x}_{2}\right)=\mu\left(\mathrm{y}_{1}, \mathrm{y}_{2}\right)=1 / 4 .
$$

That is, the unique correlated equilibrium is the Nash equilibrium in which each player independently randomizes among his two strategies, choosing each 
with probability $1 / 2$. In this unique equilibrium, each player is indifferent between his two pure strategies, and so Matching Pennies is not an elementary game (even though this equilibrium actually is perfect).

To reduce Matching Pennies to an elementary game, we must acknowledge an inextricable connection between each player's two strategies and somehow consolidate them. The key to this reduction is in the interpretation that gives rise to the game's title "Matching Pennies." Suppose that strategies $x_{i}$ and $y_{i}$ are interpreted respectively as "put i's penny Heads-up" and "put i's penny Tails-up." Player 2 pays a dollar to player 1 if their pennies match, but player 1 pays a dollar to player 2 if their pennies do not match. Then we could give either player the advice to forget about choosing Heads or Tails and simply toss the penny, so that it is equally likely to fall Heads or Tails. In effect, this advice tells player $i$ to consolidate the strategies $x_{i}$ and $y_{i}$ into a single randomized strategy that gives probability $1 / 2$ to $x_{i}$ and probability $1 / 2$ to $\mathrm{y}_{\mathrm{i}}$. After such consolidation, when each player $i$ considers only this randomized strategy $.5\left[\mathrm{x}_{i}\right]+.5\left[\mathrm{y}_{i}\right]$, we are left with a $1 \times 1$ game (with expected payoff 0 for each player) which trivially satisfies the conditions of being elementary.

These examples illustrate the two general ways that nonelementary games can be reduced: by eliminating strategies that "bad" in some sense, and by consolidating strategies that are "inextricably connected" in some sense. Elimination of dominated strategies is a familiar idea; but the consolidation of inextricably connected strategies may be less familiar. Our goal here is to show how reductions of both kinds can be derived from a unified theory of dual reduction. 
3. Duality and the existence of correlated equilibria

The existence of correlated equilibria for finite games was known since the concept was first defined by Aumann (1974), because correlated equilibria include all Nash equilibria, and Nash (1951) proved the general existence of Nash equilibria for all finite strategic-form games. Nash's proof relies on fixed-point theorems of algebraic topology, however. Hart and schmeidler (1989) and Nau and McCardle (1990) directly proved the existence of correlated equilibria using the duality theorems of linear algebra. ${ }^{1}$ In this section, we review some key ideas from Nau and McCardle's proof. Their techniques for proving existence of correlated equilibria will also be useful for showing how to reduce nonelementary games.

The first step in the proof is to set up a linear programing problem for which the optimal solutions are the correlated equilibria. There are several ways to do this, but the most useful formulation for our purposes is the following linear programming problem, which we may call the strategic incentive problem:

$$
\begin{aligned}
& \text { choose } \mu \text { and } \nu \text { to maximize } \sum_{i \in N} \Sigma_{c_{i} \in C_{i}} \nu_{i}\left(c_{i}\right) \\
& \text { subject to: } \\
& \mu(c) \geq 0 \quad \forall c \in C, \\
& \sum_{c \in C} \mu(c)=1, \text { and } \\
& \sum_{c-i} \in C-i
\end{aligned}
$$

${ }^{1}$ Nash (1951) remarked that a finite game with rational utility numbers might have only equilibria with irrational probabilities. The linear algebraic proof guarantees that such a finite game with rational utility numbers must have a correlated equilibrium in which all probabilies are rational numbers. 
The constraints of this strategic incentive problem differ from the definition of correlated equilibrium only in that we have added the artificial variables $\nu_{i}\left(c_{i}\right)$, so that feasibility of the problem can be trivially guaranteed by letting these unconstrained artificial variables become negative. Notice, however, that the vector $\nu$ must satisfy $\nu_{i}\left(c_{i}\right) \leq 0, \quad \forall i \in N, \quad \forall c_{i} \in C_{i}$, because $d_{i}=c_{i}$ is allowed in the last row of the strategic incentive problem. Furthermore, these nonpositive $\nu_{i}\left(c_{i}\right)$ numbers can al1 equal zero (their maximum possible value) if and only if $\mu$ is a correlated equilibrium. Thus, the existence of correlated equilibria can be proven by showing that the optimal value of this strategic incentive problem is zero.

Like any linear programming problem, this strategic incentive problem is associated with a dual problem that has the same optimal value, by the duality theorem of linear programming (see Chvatal, 1983, for example). Following Nau and McCardle (1990), we can formulate this dual problem and show directly that its optimal value is 0 .

The dual problem has one decision variable for each constraint in the primal. We let $\alpha_{i}\left(d_{i} \mid c_{i}\right)$ denote the dual variable associated with the primal constraint

$$
\Sigma_{c_{-i} \in C_{-i}} \mu(c)\left(U_{i}\left(c_{-i}, d_{i}\right)-U_{i}(c)\right)+\nu_{i}\left(c_{i}\right) \leq 0,
$$

which is just a relaxed version of the incentive constraint that player $i$ should not expect to gain by using $d_{i}$ when $c_{i}$ is recommended. We may let $\alpha$ denote the vector of these dual variables, and let $\alpha_{i}$ denote the subvector of components indexed on player i's strategies; that is:

$$
\alpha_{i}=\left(\alpha_{i}\left(d_{i} \mid c_{i}\right)\right)_{c_{i} \in C_{i}, d_{i} \in C_{i}}
$$




$$
\alpha=\left(\alpha_{i}\right)_{i \in \mathbb{N}}=\left(\alpha_{i}\left(d_{i} \mid c_{i}\right)\right)_{c_{i} \in C_{i}, d_{i} \in C_{i}, i \in N}
$$

Let $\beta$ denote the dual variable associated with the primal constraint $\Sigma_{c \in C} \mu(c)=1$. Then the dual problem may be written:

$$
\begin{aligned}
& \text { choose } \alpha \text { and } \beta \text { to minimize } \beta \\
& \text { subject to: } \\
& \alpha_{i}\left(d_{i} \mid c_{i}\right) \geq 0, \forall i \in N, \forall c_{i} \in C_{i}, \forall d_{i} \in C_{i}, \\
& \sum_{d_{i} \in C_{i}} \alpha_{i}\left(d_{i} \mid c_{i}\right)=1, \forall c_{i} \in C_{i}, \forall i \in N, \\
& \sum_{i \in N} \sum_{d_{i} \in C_{i}} \alpha_{i}\left(d_{i} \mid c_{i}\right)\left(U_{i}\left(c_{-i}, d_{i}\right)-U_{i}(c)\right)+\beta \geq 0, \quad \forall c \in C .
\end{aligned}
$$

Nau and McCardle (1990) have discussed the possible interpretations of these dual variables. Here we review one useful interpretation.

A strategic transition matrix for player $i$ is any $\alpha_{i}$ such that

$$
\begin{aligned}
& \alpha_{i}=\left(\alpha_{i}\left(d_{i} \mid c_{i}\right)\right)_{c_{i} \in C_{i}, d_{i} \in C_{i}} \\
& \alpha_{i}\left(d_{i} \mid c_{i}\right) \geq 0, \quad \forall c_{i} \in C_{i}, \quad \forall d_{i} \in C_{i}, \\
& \sum_{d_{i} \in C_{i}} \alpha_{i}\left(d_{i} \mid c_{i}\right)=1, \quad \forall c_{i} \in C_{i}, \quad \forall i \in N .
\end{aligned}
$$

Any such strategic transition matrix for player $i$ may be interpreted as a random deviation strategy for player $i$ in the game where a mediator is trying to implement some correlated strategy $\mu$. In this interpretation of $\alpha_{i}$, each number $\alpha_{i}\left(\alpha_{i} \mid c_{i}\right)$ represents the probability that player $i$ would deviate to the strategy $d_{i}$ when $c_{i}$ is recommended by the mediator.

Mathematically, any strategic transition matrix $\alpha_{i}$ for player $i$ can also be viewed as the matrix of transition probabilities for a Markov chain on the set of pure strategies for player i. By the basic theory of Markov chains, there must exist a probability distribution on $C_{i}$ that is stationary under this 
Markov chain. So let us say that a distribution $\sigma_{i}$ in $\Delta\left(C C_{i}\right)$ is $\underline{\alpha}_{i}$-stationary iff

$$
\Sigma_{c_{i} \in C_{i}} \sigma_{i}\left(c_{i}\right) \alpha_{i}\left(d_{i} \mid c_{i}\right)=\sigma_{i}\left(d_{i}\right), \quad \forall d_{i} \in C_{i} .
$$

As a probability distribution over $C_{i}$, any such $\alpha_{i}$-stationary distribution can also be interpreted as a randomized strategy for player i. If a mediator's recommendations to player $i$ were generated according to such an $\alpha_{i}$-stationary randomized strategy, then applying the strategic transition matrix $\alpha_{i}$ would not change the distribution of player i's actions.

For any correlated strategy $\mu$, let $\mathrm{U}_{i}(\mu)$ denote the expected payoff to player $i$ under the correlated strategy $\mu$; that is,

$$
\mathrm{U}_{\mathrm{i}}(\mu)=\Sigma_{\mathrm{c} \in \mathrm{C}} \mu(\mathrm{c}) \mathrm{U}_{\mathrm{i}}(\mathrm{c}) .
$$

Given any correlated strategy $\mu$ and strategic transition matrix $\alpha_{i}$, let $\alpha_{i} * \mu$ denote the correlated strategy that results when a mediator tries to implement the correlated strategy $\mu$, but player $i$ follows the random deviation strategy $\alpha_{i}$ while everyone else follows the mediator's recommendations. That is, $\alpha_{i} * \mu$ is the correlated strategy such that

$$
\left(\alpha_{i} * \mu\right)(\mathrm{d})=\Sigma_{c_{i} \in C_{i}} \alpha_{i}\left(d_{i} \mid c_{i}\right) \mu\left(d_{-i}, c_{i}\right), \quad \forall d \in C .
$$

Let $D_{i}\left(c, \alpha_{i}\right)$ denote the gains to player $i$ from using the random deviation strategy $\alpha_{i}$ when the mediator recommends the pure-strategy profile c. That is, let

$$
D_{i}\left(c, \alpha_{i}\right)=\Sigma_{d_{i} \in C_{i}} \alpha_{i}\left(d_{i} \mid c_{i}\right)\left(U_{i}\left(c_{-i}, d_{i}\right)-U_{i}(c)\right) .
$$

With this notation, we may write the following identity, when $\mu$ is any correlated strategy $\mu$ in $\Delta(C)$, and $\alpha_{i}$ is any strategic transition matrix for 
player i.

$$
\Sigma_{c \in C} \mu(c) D_{i}\left(c, \alpha_{i}\right)=U_{i}\left(\alpha_{i} * \mu\right)-U_{i}(\mu) .
$$

To verify (3), notice that both sides of the equation represent the expected gains for player $i$ from deviating by $\alpha_{i}$, when all other players obey a mediator who is trying to implement the correlated strategy $\mu$.

Thus, the dual problem requires us to specify a profile of random deviation strategies $\alpha=\left(\alpha_{i}\right)_{i \in N}$ and a number $\beta$ such that

$$
\beta+\Sigma_{i \in N} D_{i}\left(c, \alpha_{i}\right) \geq 0, \quad \forall c \in C .
$$

For any $(\alpha, \beta)$ that satisfies these dual constraints, and for any correlated strategy $\mu$ in $\Delta(C)$, we must have

$$
0 \leq \beta+\Sigma_{c \in C} \mu(c) \Sigma_{i \in N} D_{i}\left(c, \alpha_{i}\right)=\beta+\Sigma_{i \in N}\left(U_{i}(\mu)-U_{i}\left(\alpha_{i} * \mu\right)\right) .
$$

But now consider a correlated strategy that is constructed by telling each player $i$ to independently randomize over $C_{i}$ according to a strategy that is $\alpha_{i}$-stationary. That is, consider $\mu$ such that

$$
\mu(c)=\Pi_{i \in N} \sigma_{i}\left(c_{i}\right), \forall c \in C,
$$

where each randomized strategy $\sigma_{i}$ satisfies the ${ }^{\prime}{ }_{i}$-stationarity condition (2). Then $\alpha_{i}{ }^{*} \mu=\mu$ for every player $i$, and so we get simply

$$
0 \leq \beta \text {. }
$$

That is, a feasible solution for the dual problem cannot have a negative value for the objective. So the optimal value of the objective $\beta$ in the dual problem must be zero. (Notice that $\beta=0$ can be achieved trivially by letting $\alpha_{i}\left(d_{i} \mid c_{i}\right)=0$ whenever $\left.d_{i} \neq c_{i}.\right)$ So the optimal value of the strategic incentive problem is also zero, by the duality theorem of linear programming. This observation completes the linear-algebraic proof of the general existence of correlated equilibria.

Given the existence of correlated equilibria, we know that any optimal 
solution of the dual problem must have $\beta=0$, and the vector $\alpha$ must satisfy

$$
\begin{aligned}
& \alpha_{i}\left(d_{i} \mid c_{i}\right) \geq 0, \quad \forall i \in N, \quad \forall c_{i} \in C_{i}, \quad \forall d_{i} \in C_{i} \\
& \sum_{d_{i} \in C} \alpha_{i}\left(d_{i} \mid c_{i}\right)=1, \quad \forall c_{i} \in C_{i}, \quad \forall i \in N, \quad \text { and } \\
& \sum_{i \in N} \sum_{d_{i} \in C_{i}} \alpha_{i}\left(d_{i} \mid c_{i}\right)\left(U_{i}\left(c_{-i}, d_{i}\right)-U_{i}(c)\right) \geq 0, \forall c \in C .
\end{aligned}
$$

So henceforth let us say that $\alpha$ is a dual vector for the game $\Gamma$ iff $\alpha$ satisfies these conditions (which are the dual constraints with $\beta=0$ ).

\section{Dua1 reductions}

We have seen that a dual vector can be interpreted as a profile of Markov chains on the players' pure strategy sets. The fact that stationary distributions exist for the these Markov chains was the key step in proving that the dual problem has optimal value 0 , which in turn implied that correlated equilibria must exist. That is, in a quest to find correlated equilibria, we found dual-stationary strategies first, and then we found the equilibria. This derivation suggests that these stationary strategies might form some kind of pre-equilibrium solution concept for games with communication. Following this intuition, let us see how a game is transformed when the players restrict themselves to randomized strategies that are stationary with respect to some dual vector $\alpha=\left(\alpha_{i}\right)_{i \in N}$.

First we must review some of the basic results from the theory of Markov chains, as they apply when we consider each $\alpha_{i}$ as a Markov chain on $C_{i}$. We may say that a set $B_{i}$ is an $\underline{\alpha}_{i}$-absorbing iff $B_{i}$ is a nonempty subset of $C_{i}$ and

$$
\alpha_{i}\left(c_{i} \mid b_{i}\right)=0, \quad \forall b_{i} \in B_{i}, \quad \forall c_{i} \in C_{i} \backslash B_{i}
$$

That is, $B_{i}$ is $\alpha_{i}$-absorbing iff $\alpha_{i}$ assigns zero probability to moves outside the set $B_{i}$ from within $B_{i}$. A minimal $\alpha_{i}$-absorbing set is any $\alpha_{i}$-absorbing set 
that contains no proper $\alpha_{i}$-absorbing subset. Because $C_{i}$ is finite, at least one such minimal $\alpha_{i}$-absorbing set must exist. From the basic theory of Markov chains (see Karlin, 1969, for example), if $B_{i}$ is any minimal $\alpha_{i}$-absorbing set then there exists a unique $\alpha_{i}$-stationary distribution that has support on $B_{i}$. That is, for any minimal $\alpha_{i}$-absorbing subset $B_{i}$, there is a unique randomized strategy $\sigma_{i}$ such that

$$
\begin{aligned}
& \Sigma_{c_{i} \in B_{i}} \sigma_{i}\left(c_{i}\right) \alpha_{i}\left(b_{i} \mid c_{i}\right)=\sigma_{i}\left(b_{i}\right), \quad \forall b_{i} \in B_{i}, \\
& \Sigma_{c_{i} \in B_{i}} \sigma_{i}\left(c_{i}\right)=1, \quad \text { and } \\
& \sigma_{i}\left(d_{i}\right)=0 \quad \forall d_{i} \notin B_{i} .
\end{aligned}
$$

Let $C_{i} / \alpha_{i}$ denote the set of these $\alpha_{i}$-stationary distributions that have support within a minimal $\alpha_{i}$-absorbing subset of $\mathrm{C}_{i} \cdot\left(\mathrm{C}_{i} / \alpha_{i}\right.$ may be read as "C $i$ reduced by $\alpha_{i}$ ") Notice that $\mathrm{C}_{i} / \alpha_{i}$ is a nonempty finite subset of $\Delta\left(\mathrm{C}_{i}\right)$, the set of randomized strategies for player $i$. In fact, the number of elements in this set $C_{i} / \alpha_{i}$ cannot be greater than the number of pure strategies in $C_{i}$, because there is one element of $\mathrm{C}_{i} / \alpha_{i}$ for every minimal $\alpha_{i}$-absorbing set, and these minimal $\alpha_{i}$-absorbing sets are nonempty disjoint subsets of $C_{i}$.

Other $\alpha_{i}$-stationary strategies can be constructed by taking convex combinations of these randomized strategies in $\mathrm{C}_{i} / \alpha_{i}$, but it can be shown that these are all the $\alpha_{i}$-stationary strategies. That is, any $\alpha_{i}$-stationary randomized strategy in $\Delta\left(C_{i}\right)$ must be equivalent to a random mixture of the strategies in this finite set $C_{i} / \alpha_{i}$. Geometrically, the distributions in $C_{i} / \alpha_{i}$ are the extreme points of the set of all $\alpha_{i}$-stationary distributions on $C_{i}$. So let us define the $\alpha$-reduction of $\Gamma$, denoted by $\Gamma / \alpha$, to be the game in which every player restricts himself to using $\alpha_{i}$-stationary strategies. In this game $\Gamma / \alpha$, the set of players is $N$ (as in $\Gamma$ ), but the set of pure 
strategies for each player $i$ is $C_{i} / \alpha_{i}$, instead of $C_{i}$. For any profile of strategies $\sigma=\left(\sigma_{i}\right)_{i \in N}$ in $x_{i \in N} C_{i} / \alpha_{i}$, the utility function in $\Gamma / \alpha$ is simply $u_{i}(\sigma)=\Sigma_{c \in C}\left(\Pi_{j \in N} \sigma_{i}\left(c_{i}\right)\right) U_{i}(c)$.

This equation asserts that, once player $i$ has chosen an $\alpha_{i}$-stationary strategy $\sigma_{i}$ in $C_{i} / \alpha_{i}$, any randomization demanded by $\sigma_{i}$ is implemented independently of the other players. Notice that this $\alpha$-reduced game $\Gamma / \alpha$ is itself a finite strategic-form game, with the structure

$$
\Gamma / \alpha=\left(N, \quad\left(C_{i} / \alpha_{i}\right){ }_{i \in N},\left(u_{i}\right)_{i \in N}\right) .
$$

More generally, we may use the term dual reduction to refer to any such reduced game that can be derived from $\Gamma$ using any dual vector of $\Gamma$.

Let $\mathrm{C} / \alpha=\times_{i \in \mathrm{N}} \mathrm{C}_{i} / \alpha_{i}$ denote the set of strategy profiles in the $\alpha$-reduced game $\Gamma / \alpha$. So the set of correlated strategies in the $\alpha$-reduced game is $\Delta(C / \alpha)$. But any $\lambda$ in $\Delta(C / \alpha)$ can be mapped back to a $\Gamma$-equivalent correlated strategy $\bar{\lambda}$ in $\Delta(C)$ in the obvious way:

$$
\bar{\lambda}(c)=\Sigma_{\sigma \in \mathrm{C} / \alpha}\left(\Pi_{i \in \mathbb{N}} \sigma_{i}\left(c_{i}\right)\right) \lambda(\sigma) .
$$

Thus, when we assume that the players use strategies that are stationary with respect to some dual vector $\alpha$, we get a reduced game $\Gamma / \alpha$, and we can analyze the equilibria of this reduced game like any other finite strategic-form game. But now we must ask whether it would actually be rational for the players to act according to an equilibrium of the $\alpha$-reduced game, once we admit that any player could also deviate to a nonstationary strategy. That is, when we find an equilibrium in the $\alpha$-reduced game, is it also an equilibrium in the original game? The answer to this question is Yes. This result gives us our fundamental justification for studying dual reductions, and it is the main result of this paper. Its proof is given in Section 8 . 
Theorem 1. Let $\alpha$ be a dual vector for the game $\Gamma$. If $\lambda$ is any correlated equilibrium of the $\alpha$-reduced game $\Gamma / \alpha$, then the $\Gamma$-equivalent correlated strategy $\dot{\lambda}$ is also a correlated equilibrium of $\Gamma$.

\section{Iterative dual reduction and elementary games}

By Theorem 1, the equilibria of a dual reduction $\Gamma / \alpha$ form a nonempty subset of the equilibria of the original game $\Gamma$. The game $\Gamma$ may have other equilibria of $\Gamma$ that do not correspond to any equilibria of $\Gamma / \alpha$, however. Thus, dual reduction may be viewed as a tool for refining the set of equilibria.

But a dual reduction is itself another strategic-form game that may be further reducible, which can give us a further refinement of the equilibrium set. So we may say that an iterative dual reduction of $\Gamma$ is any $\Gamma / \alpha^{1} / \alpha^{2} / \ldots$ where each $\alpha^{\mathrm{K}}$ is a dual vector for $\Gamma / \alpha^{1} / \alpha^{2} / \ldots / \alpha^{K-1}$. Repeated application of Theorem 1 assures us that equilibrium inclusion holds for iterative dual reduction. That is, any equilibrium (correlated or Nash) of any game that can be generated from $\Gamma$ by iterative dual reduction must be an equilibrium of the original game $\Gamma$.

So we should now ask, what do we get from applying iterative dual reduction until we reach a game that cannot be further reduced? We now show that the result will be an elementary game, as defined in Section 2 .

We may say that a dual vector $\alpha$ is trivial iff

$$
\alpha_{i}\left(c_{i} \mid c_{i}\right)=1 \quad \text { and } \alpha_{i}\left(d_{i} \mid c_{i}\right)=0, \quad \forall d_{i} \neq c_{i}, \quad \forall c_{i} \in C_{i}, \quad \forall i \in N .
$$

That is, the trivial dual vector specifies that every player always obeys the mediator's recommendations with probability one. This trivial dual vector always satisfies the dual constraints (with $\beta=0$ ), because it sets all 
deviation gains $\mathrm{D}_{\mathrm{i}}\left(c, \alpha_{i}\right)$ equal to zero.

We have already observed that, for any dual vector $\alpha$, number of "pure" strategies for each player in the reduced game $\Gamma / \alpha$ is not more than the number of pure strategies that this player has in the original game $\Gamma$. That is, letting $\#(S)$ denote the number of elements in any set $S$, we may write

$$
\#(C / \alpha)=\Pi_{i \in N} \#\left(C_{i} / \alpha_{i}\right) \leq \Pi_{i \in N} \#\left(C_{i}\right)=\#(C) .
$$

This inequality can be made strict if and only if $\alpha$ is nontrivial. If $\alpha_{i}\left(d_{i} \mid c_{i}\right)>0$ and $d_{i} \neq c_{i}$, then either $c_{i}$ must be grouped together with $d_{i}$ in the same minimal absorbing set or else $c_{i}$ must be a transient strategy that is not included in any minimal $\alpha_{i}$-absorbing set. So if $\alpha$ is any nontrivial dual vector then $\alpha$-reduction generates a strict decrease in the (finite) number of pure-strategy profiles

$$
\#(C / \alpha)<\#(C) \text {. }
$$

Conversely, the trivial dual vector gives no reduction, because every strategy in $C_{i}$ is stationary under the trivial dual vector.

Thus, dual reduction can be iteratively applied to generate strictly smaller games until we reach a game that has no nontrivial dual vector. Propositions 2 and 3 tell us when such nontrivial dual vectors can be found.

Proposition 2. For player $j$, let $b_{j}$ and $e_{j}$ be any two pure strategies in $C_{j}$, where $b_{j} \neq e_{j}$. Then there exists a dual vector $\alpha$ such that

$$
\alpha_{j}\left(b_{j} \mid e_{j}\right)>0
$$

if and only if, for every correlated equilibrium $\mu$,

$$
\Sigma_{e_{-j} \in C_{-j}} \mu(e)\left(U_{j}\left(e_{-j}, b_{i}\right)-U_{i}(e)\right)=0 .
$$

That is, the dual variable $\alpha_{j}\left(b_{j} \mid e_{j}\right)$ can be nonzero if and only if the incentive constraint that player $j$ should not expect to gain by choosing $b_{j}$ 
when $e_{j}$ is recommended is always binding. If this constraint is always binding then we may say that the strategy $b_{j}$ jeopardizes $e_{j}$ for player $j$.

Proposition 2 is a straightforward application of the complementary slackness properties of dual linear programs. Its proof is given Section 8 . By Proposition 2, if $\Gamma$ is an elementary game then there are no incentive constraints that always bind, and so the only dual vector is the trivial one. However, if $\Gamma$ is not an elementary game, then there exists at least one incentive constraint that is always binding, and so Proposition 2 guarantees that a nontrivial dual vector must exist. So Proposition 2 has the following corollary.

Proposition 3. A nontrivial dual vector $\alpha$ exists for the game $\Gamma$ if and only if $\Gamma$ is not elementary.

Thus, dual reduction can be applied iteratively until we get to an elementary game, which must occur eventually bec ause each nontrivial dual reduction strictly decreases the (finite) number of pure-strategy profiles. This basic result should be listed as our second theorem, because it suggests that game theorists may study elementary games without loss of generality.

Theorem 2. From any finite strategic-form game $\Gamma$, an elementary game can be derived by iterative dual reduction.

\section{Examples}

Recall that any dual vector must satisfy the constraints

$$
\Sigma_{i \in N} D_{i}\left(c, \alpha_{i}\right)=\Sigma_{i \in N} \Sigma_{d_{i} \in C} \alpha_{i}\left(d_{i} \mid c_{i}\right)\left(U_{i}\left(c_{-i}, d_{i}\right)-U_{i}(c)\right) \geq 0, \quad \forall c \in C .
$$

(Here the first equality merely repeats the definition of $D_{i}\left(c, \alpha_{i}\right)$. ) 
Dual reduction includes, as a special case, the elimination of dominated strategies. To verify this fact, suppose that a pure strategy $e_{j}$ is (weakly) dominated by some randomized strategy $\tau_{j}$ for player $j$. Then

$$
U_{j}(e) \leq \Sigma_{d_{j} \in C_{j}} \tau_{j}\left(d_{j}\right) U_{j}\left(e_{-j}, d_{j}\right), \quad \forall e_{-i} \in C_{-i} .
$$

In this case, we can let $\alpha$ be such that

$$
\begin{aligned}
& \alpha_{j}\left(d_{j} \mid e_{j}\right)=\tau_{j}\left(d_{j}\right), \quad \forall d_{j} \in c_{j}, \\
& \alpha_{i}\left(c_{j} \mid c_{j}\right)=1 \text { and } \alpha_{j}\left(d_{j} \mid c_{j}\right)=0 \quad \forall d_{j} \neq c_{j}, \quad \forall c_{j} \neq e_{j}, \\
& \alpha_{i}\left(c_{i} \mid c_{i}\right)=1 \text { and } \alpha_{i}\left(d_{i} \mid c_{i}\right)=0 \quad \forall d_{i} \neq c_{i}, \forall c_{i} \in c_{i}, \forall i \neq j .
\end{aligned}
$$

With this vector $\alpha$, the deviation gains $D_{j}\left(e, \alpha_{j}\right)$ gains are nonnegative for all $e_{-j}$, and all other deviation gains $D_{i}\left(c, \alpha_{i}\right)$ are zero. Thus, $\alpha$ is a dual vector satisfying the dual constraints (4). Under the Markov chains induced by this dual vector $\alpha$, every pure strategy of every player is stationary, except for $e_{j}$, which is a transient strategy that must get probability zero in any $\alpha_{j}$-stationary strategy. So $\Gamma / \alpha$ is just the game that results from eliminating the strategy $e_{i}$.

Player 2

$\begin{array}{rrrrc} & & \mathrm{x}_{2} & \mathrm{y}_{2} & \mathrm{z}_{2} \\ \text { Player 1 } & \mathrm{x}_{1} & 3,2 & 0,0 & 0,1 \\ & \mathrm{y}_{1} & 0,0 & 2,3 & 3,1\end{array}$

Figure 4. The Battle of the Sexes with a dominated strategy.

For example, consider the game shown in Figure 4. For any number $\delta$ between $1 / 2$ and $2 / 3$, we can construct a dual vector by letting

$$
\alpha_{2}\left(x_{2} \mid z_{2}\right)=\delta, \quad \alpha_{2}\left(y_{2} \mid z_{2}\right)=1-\delta, \quad \alpha_{2}\left(z_{2} \mid z_{2}\right)=0,
$$


and by letting all other components of $\alpha$ be as in the trivial dual vector. With this dual vector $\alpha$, the deviation gains $D_{i}\left(c, \alpha_{i}\right)$ are positive for the case of $i=2$ and $c_{2}=z_{2}$, and they are zero in all o:her cases, so the dual constraints (4) are satisfied. This dual vector eliminates the dominated strategy $z_{2}$ from the $\alpha$-reduced game. So the $\alpha$-reduced game is just the Battle of the Sexes game in Figure 1, which is elementary and so cannot be further reduced.

More generally, a dual vector can be constructed by letting each $\alpha_{i}$ be a strategic transition matrix which maps each dominated pure strategy to a randomized strategy that dominates it, and which leaves all undominated pure strategies fixed. For the Prisoners' Dilemma game in Figure 2, this approach gives us the dual vector $\alpha$ such that

$$
\alpha_{1}\left(y_{1} \mid x_{1}\right)=1, \quad \alpha_{1}\left(y_{1} \mid y_{1}\right)=1, \quad \alpha_{2}\left(y_{2} \mid z_{2}\right)=1, \quad \alpha_{2}\left(y_{2} \mid y_{2}\right)=1,
$$

which eliminates the two dominated strategies.

Player 2

$\begin{array}{cccc} & & x_{2} & y_{2} \\ \text { Player 1 } & x_{1} & 7,0 & 2,5 \\ & y_{1} & 4,3 & 6,1\end{array}$

Figure 5. A constant-sum game with a unique randomized equilibrium.

Now consider the game shown in Figure 5. Like the Matching Pennies game, this game has a unique randomized equilibrium

$$
\left(\frac{2}{7}\left[\mathrm{x}_{1}\right]+\frac{5}{7}\left[\mathrm{y}_{1}\right], \frac{4}{7}\left[\mathrm{x}_{2}\right]+\frac{3}{7}\left[\mathrm{y}_{2}\right]\right)
$$

in which each player is indifferent between his two pure strategies. For this game, the dual constraints (4) become: 


$$
\begin{aligned}
& \alpha_{1}\left(y_{1} \mid x_{1}\right)(4-7)+\alpha_{2}\left(y_{2} \mid x_{2}\right)(5-0) \geq 0 \quad\left(\text { at } c=\left(x_{1}, x_{2}\right)\right), \\
& \alpha_{1}\left(y_{1} \mid x_{1}\right)(6-2)+\alpha_{2}\left(x_{2} \mid y_{2}\right)(0-5) \geq 0 \quad\left(\text { at } c=\left(x_{1}, y_{2}\right)\right), \\
& \alpha_{1}\left(x_{1} \mid y_{1}\right)(2-6)+\alpha_{2}\left(x_{2} \mid y_{2}\right)(3-1) \geq 0 \quad\left(\text { at } c=\left(y_{1}, y_{2}\right)\right), \\
& \alpha_{1}\left(x_{1} \mid y_{1}\right)(7-4)+\alpha_{2}\left(y_{2} \mid x_{2}\right)(1-3) \geq 0 \quad\left(\text { at } c=\left(y_{1}, x_{2}\right)\right),
\end{aligned}
$$

Together these constraints imply

$$
5 \alpha_{2}\left(y_{2} \mid x_{2}\right) \geq 3 \alpha_{1}\left(y_{1} \mid x_{1}\right) \geq(15 / 4) \alpha_{2}\left(x_{2} \mid y_{2}\right) \geq(15 / 2) \alpha_{1}\left(x_{1} \mid y_{1}\right) \geq 5 \alpha_{2}\left(y_{2} \mid x_{2}\right),
$$

and so all these quantities are equal. Thus

$$
\alpha_{1}\left(y_{1} \mid x_{1}\right) / \alpha_{1}\left(x_{1} \mid y_{1}\right)=5 / 2 \text { and } \alpha_{2}\left(y_{2} \mid x_{2}\right) / \alpha_{2}\left(x_{2} \mid y_{2}\right)=3 / 4
$$

But $\alpha_{i}$-stationary strategies $\sigma_{i}$ must satisfy

$$
\begin{aligned}
& \alpha_{1}\left(\mathrm{y}_{1} \mid \mathrm{x}_{1}\right) \sigma_{1}\left(\mathrm{x}_{1}\right)=\alpha_{1}\left(\mathrm{x}_{1} \mid \mathrm{y}_{1}\right) \sigma_{1}\left(\mathrm{y}_{1}\right), \\
& \alpha_{2}\left(\mathrm{y}_{2} \mid \mathrm{x}_{2}\right) \sigma_{2}\left(\mathrm{x}_{2}\right)=\alpha_{2}\left(\mathrm{x}_{2} \mid \mathrm{y}_{2}\right) \sigma_{2}\left(\mathrm{y}_{2}\right) .
\end{aligned}
$$

So a nontrivial dual vector can only have the stationary strategies

$$
\sigma_{1}\left(\mathrm{x}_{1}\right)=2 / 7, \sigma_{1}\left(\mathrm{y}_{1}\right)=5 / 7, \quad \sigma_{2}\left(\mathrm{x}_{2}\right)=4 / 7, \sigma_{2}\left(\mathrm{y}_{2}\right)=3 / 7,
$$

which is of course the unique equilibrium.

(Similarly, for the Matching Pennies game in Figure 3, it can be shown that any dual vector $\alpha$ must satisfy $\alpha_{1}\left(\mathrm{y}_{1} \mid \mathrm{x}_{1}\right)=\alpha_{1}\left(\mathrm{x}_{1} \mid \mathrm{y}_{1}\right)=\alpha_{2}\left(\mathrm{y}_{2} \mid \mathrm{x}_{2}\right)=$ $\alpha_{2}\left(y_{2} \mid x_{2}\right)$. But then any nontrivial dual vector $\alpha$ can only have one stationary distribution $\sigma_{i}$ for each player $i$, and that is the equilibrium strategy that puts probability $1 / 2$ on each pure strategy.)

Figures 6 and 7 show two games in which no pure strategies are dominated, and yet dual reduction can simplify the game and eliminate some equilibria. For Figure 6, let $\alpha$ be such that

$$
\begin{aligned}
& \alpha_{1}\left(x_{1} \mid x_{1}\right)=\alpha_{1}\left(x_{1} \mid y_{1}\right)=\alpha_{1}\left(x_{1} \mid z_{1}\right)=1, \\
& \alpha_{2}\left(x_{2} \mid x_{2}\right)=\alpha_{2}\left(x_{2} \mid y_{2}\right)=\alpha_{2}\left(x_{2} \mid z_{2}\right)=1,
\end{aligned}
$$

and all other $\alpha_{i}\left(d_{i} \mid c_{i}\right)=0$. This dual vector $\alpha$ satisfies the dual constraints (4), and it eliminates the strategies $y_{1}, z_{1}, y_{2}$, and $z_{2}$ from the $\alpha$-reduced 
game. Thus, the equilibrium $\left(.5\left[y_{1}\right]+.5\left[z_{1}\right],\left[x_{2}\right]\right)$ can be eliminated by dual reduction.

\begin{tabular}{ccccc} 
& \multicolumn{5}{c}{ Player 2} \\
Player 1 & \multicolumn{5}{c}{$\mathrm{x}_{2}$} & $\mathrm{y}_{2}$ & $\mathrm{z}_{2}$ \\
& $\mathrm{x}_{1}$ & 2,2 & 4,2 & 4,2 \\
& $\mathrm{y}_{1}$ & 2,4 & 6,0 & 0,6 \\
& $z_{1}$ & 2,4 & 0,6 & 6,0
\end{tabular}

Figure 6. A game with no dominated pure strategies.

Player 2

$\begin{array}{rcccc} & & \mathrm{x}_{2} & \mathrm{y}_{2} & \mathrm{z}_{2} \\ \text { Player 1 } & \mathrm{w}_{1} & 6,0 & 0,0 & 0,0 \\ & \mathrm{x}_{1} & 4,4 & 1,4 & 4,1 \\ \mathrm{y}_{1} & 4,1 & 4,4 & 1,4 \\ \mathrm{z}_{1} & 1,4 & 4,1 & 4,4\end{array}$

Figure 7. Another game with no dominated pure strategies.

For Figure 7, let $\alpha$ be such that

$$
\begin{aligned}
& \alpha_{1}\left(y_{1} \mid x_{1}\right)=\alpha_{1}\left(z_{1} \mid y_{1}\right)=\alpha_{1}\left(x_{1} \mid z_{1}\right)=1=\alpha_{1}\left(w_{1} \mid w_{1}\right), \\
& \alpha_{2}\left(y_{2} \mid x_{2}\right)=\alpha_{2}\left(z_{2} \mid y_{2}\right)=\alpha_{2}\left(x_{2} \mid z_{2}\right)=1,
\end{aligned}
$$

and all other $\alpha_{i}\left(d_{i} \mid c_{i}\right)=0$. This dual vector $\alpha$ satisfies the dual constraints (4). It leaves player 1 with two extreme stationary strategies

$$
\left[\mathrm{w}_{1}\right], \text { and } \sigma_{1}=\frac{1}{3}\left[\mathrm{x}_{1}\right]+\frac{1}{3}\left[\mathrm{y}_{1}\right]+\frac{1}{3}\left[\mathrm{z}_{1}\right] \text {, }
$$

and it leaves player 2 with only one stationary strategy

$$
\sigma_{2}=\frac{1}{3}\left[x_{2}\right]+\frac{1}{3}\left[y_{2}\right]+\frac{1}{3}\left[z_{2}\right],
$$


Then the $\alpha$-reduced game is as shown in Figure 8 .

Player 2

$\begin{array}{ccc} & & \sigma_{2} \\ \text { Player } 1 & \mathrm{w}_{1} & 2,0 \\ & \sigma_{1} & 3,3\end{array}$

Figure 8. A dual reduction of Figure 7 .

But this game can be in turn reduced by the dual vector $\hat{\alpha}$ such that

$$
\hat{\alpha}_{1}\left(\sigma_{1} \mid w_{1}\right)=\hat{\alpha}_{1}\left(\sigma_{1} \mid \sigma_{1}\right)=1=\hat{\alpha}_{2}\left(\sigma_{2} \mid \sigma_{2}\right) \text {, }
$$

which eliminates $\mathrm{w}_{1}$ and leaves us with the $\left(\sigma_{1}, \sigma_{2}\right)$ equilibrium. Thus,

iterative dual reduction can eliminate the $\left(\mathrm{w}_{1}, \mathrm{x}_{2}\right)$ equilibrium in Figure 7.

\section{Full dual reduction}

The set of dual vectors is convex, and all components are nonnegative in all dual vectors. So when we take an average of dual vectors that are positive in different components, we get a dual vector that is positive in all of these components. So we can derive the following fact from Proposition 2.

Proposition 4. There exists a dual vector $\alpha$ that satisfies

$$
\alpha_{i}\left(d_{i} \mid c_{i}\right)>0
$$

for every player $i$ and for every pair of strategies $c_{i}$ and $d_{i}$ in $c_{i}$ such that, for every correlated equilibrium $\mu, \quad \Sigma_{c_{-i} \in C} \mu(c)\left(U_{i}\left(c_{-i}, d_{i}\right)-U_{i}(c)\right)=0$.

That is, we can find a dual vector that has a positive dual variable for every incentive constraint that is binding at all correlated equilibria. Such a dual vector $\alpha$ may be said to have full support. 
We have seen that dual reduction is a powerful generalization of elimination of weakly dominated strategies. However, like elimination of weakly dominated strategies, there may be more than one way to apply dual reduction to any given game, because there may be more than one nontrivial dual vector. One possible way to avoid this ambiguity, at least in some examples, would be to only consider reduction by dual vectors that have full support. If $\alpha$ is a dual vector with full support then $\Gamma / \alpha$ may be called a full dual reduction of $\Gamma$.

In Figure 7, among player 2's pure strategies, $y_{2}$ jeopardizes $x_{2}$ (in that deviating from $\mathrm{y}_{2}$ to $\mathrm{x}_{2}$ is always costless for player 2 , in any correlated equilibrium), $z_{2}$ jeopardizes $y_{2}$, and $x_{2}$ jeopardizes $z_{2}$. So a full dual reduction of this game must consolidate these three pure strategies, replacing them by a single randomized strategy (as we saw in Figure 8). Notice, however, that $x_{2}$ does not jeopardize $y_{2}$, because deviating from $y_{2}$ to $x_{2}$ would be costly for player 2 in the $\left(y_{1}, y_{2}\right)$ equilibrium of Figure 7 .

\section{Proofs}

To prove Theorem 1, we first prove two lemmas.

Lemma 1. Let $\alpha$ be a dual vector, let $\rho_{-i}$ be in $\Delta\left(C_{-i}\right)$, and let $\sigma_{i}$ be in $\Delta\left(C_{i}\right)$. Suppose that $\alpha_{j} * \rho_{-i}=\rho_{-i}$, for every player $j$ such that $j \neq i$. Then $U_{i}\left(\rho_{-i}, \sigma_{i}\right) \leq U_{i}\left(\rho_{-i}, \alpha_{i} * \sigma_{i}\right)$.

Proof of Lemma 1. Recall equation (3) from Section 3.

$$
\begin{aligned}
0 \leq \Sigma_{c \in C} \rho_{-i}\left(c_{-i}\right) \sigma_{i}\left(c_{i}\right) \sum_{h \in N} D_{h}(c, \alpha) & \\
= & \sum_{j \in N-i}\left(U_{j}\left(\alpha_{j} * \rho_{-i}, \sigma_{i}\right)-U_{j}\left(\rho_{-i}, \sigma_{i}\right)\right)+\left(U_{i}\left(\rho_{-i}, \alpha_{i} * \sigma_{i}\right)-U_{i}\left(\rho_{-i}, \sigma_{i}\right)\right) \\
& =U_{i}\left(\rho_{-i}, \alpha_{i} * \sigma_{i}\right)-U_{i}\left(\rho_{-i}, \sigma_{i}\right) .
\end{aligned}
$$


Lemma 2: Let $\alpha$ be a dual vector, let $\rho_{-i}$ be in $\Delta\left(C_{-i}\right)$, and suppose that $\alpha_{j} \hat{p}_{-i}=\rho_{-i}$ for every player $j$ such that $j \neq i$. Then there exists some $\alpha_{i}$-stationary strategy $\sigma_{i}$ in $\mathrm{C}_{i} / \alpha_{i}$ that is a best response to $\rho_{-i}$ for player $i$, in the sense that

$$
\left.U_{i}\left(\rho_{-i}, \sigma_{i}\right)=\max _{c_{i} \in C_{i}} U_{i}\left(\rho_{-i}, c_{i}\right)\right)
$$

Proof of Lemma 2: Lemma 1 implies that, if $\tau_{i}$ is any best response to $\rho_{-i}$ for player $i$, then $\alpha_{i}{ }^{* \tau} i$ is also a best response, and so is the randomized strategy

$$
f\left(\tau_{i}\right)=\frac{1}{2} \tau i+\frac{1}{2} \alpha_{i} * \tau_{i}
$$

Now let $c_{i}$ be any best response in $C_{i}$ for player $i$, when the others are expected to use $\rho_{-i}$. Then any strategy of the form $f\left(f\left(\ldots f\left(\left[c_{i}\right]\right) \ldots\right)\right)$ is also a best response for $i$, by Lemma 1. As long as the support of this strategy is not an absorbing set, the $f(\cdot)$ transformation must strictly increase its support. So after $\#\left(C_{i}\right)$ iterations, such a process must generate best responses that assign positive probability to every pure strategy in at least one minimal $\alpha_{i}$-absorbing subset of $C_{i}$. So the $\alpha_{i}$-stationary strategy on this minimal $\alpha_{i}$-absorbing set must also be a best response for player $i$ against $\rho_{-i}$. (An similar argument, applied to a modified game where player i's options are limited to one minimal $\alpha_{i}$-absorbing set, can be used to show that player $i$ must be indifferent among all pure strategies in any minimal $\alpha_{i}$-absorbing set when the other players use such stationary $\rho_{-i}$ )

$$
\text { Q.E.D. }
$$

Proof of Theorem 1. Suppose that a mediator is implementing the correlated equilibrium $\lambda$ in the reduced game $\Gamma / \alpha$, and suppose that $\sigma_{i}$ in $C_{i} / \alpha_{i}$ has been recommended to player $i$ by this mediator. Let $\rho_{-i}$ denote the 
conditional probability distribution over the other players' strategies in $C_{-i}$, given that $\sigma_{i}$ in $C_{i} / \alpha_{i}$ has been recommended. The assumption that $\lambda$ is a correlated equilibrium for $\Gamma / \alpha$ implies that $\sigma_{i}$ must be optimal for player $i$ among all $\alpha_{i}$-stationary strategies in $\mathrm{C}_{i} / \alpha_{i}$. Furthermore, because the mediator is recommending $\alpha_{j}$-stationary strategies to every other player $j$, we must have

$$
\alpha_{j} * \rho_{-i}=\rho_{-i}, \quad \forall j \in N-i
$$

So by Lemma 2, optimality of $\sigma_{i}$ in $C_{i} / \alpha_{i}$ implies its optimality for player $i$ in all of $\Delta\left(C_{i}\right)$. That is, player $i$ cannot gain by deviating to any strategy that is available to him in $\Gamma$, and so $\bar{\lambda}$ is a correlated equilibrium of $\Gamma$.

Q.E.D.

Proof of Proposition 2. Let $\alpha$ be any dual vector and let $\mu$ be any correlated equilibrium. Then

$$
\begin{gathered}
0 \geq \Sigma_{i \in N} \Sigma_{C_{i} \in C_{i}} \Sigma_{d_{i} \in C_{i}} \alpha_{i}\left(d_{i} \mid c_{i}\right) \Sigma_{c} \in C_{-i} \mu(c)\left(U_{i}\left(c_{-i}, d_{i}\right)-U_{i}(c)\right) \\
=\Sigma_{c \in C} \mu(c) \Sigma_{i \in N} D_{i}\left(c, \alpha_{i}\right) \geq 0 .
\end{gathered}
$$

But in the above summation, each term

$$
\alpha_{i}\left(d_{i} \mid c_{i}\right) \Sigma_{c_{-i}} \in C_{-i} \mu(c)\left(U_{i}\left(c_{-i}, d_{i}\right)-U_{i}(c)\right)
$$

must be nonpositive, and so

$$
\alpha_{i}\left(d_{i} \mid c_{i}\right) \Sigma_{c-i} \in C_{-i} \mu(c)\left(U_{i}\left(c_{-i}, d_{i}\right)-U_{i}(c)\right)=0
$$

for every $i$, and for every $c_{i}$ and $d_{i}$. Thus,

$$
\text { if } \alpha_{j}\left(b_{j} \mid e_{j}\right)>0 \text { then } \Sigma_{e_{-j} \in C_{-j}} \mu(e)\left(U_{j}\left(e_{-j}, b_{j}\right)-U_{j}(e)\right)=0
$$

Now suppose that the "no incentive to choose $b_{j}$ instead of $e_{j}$ " constraint is always binding, in every correlated equilibrium. Then the following linear programming problem has an optimal value equal to zero: 


$$
\begin{aligned}
& \text { choose } \mu \text { to maximize } \Sigma_{e_{-j} \in C_{-j}} \mu(e)\left(U_{j}(e)-U_{j}\left(e_{-j}, b_{j}\right)\right) \\
& \text { subject to } \\
& \mu(c) \geq 0 \quad \forall c \in C, \\
& \Sigma_{c \in C} \mu(c)=1, \text { and } \\
& \sum_{c_{-i} \in C_{-i}} \mu(c)\left(U_{i}\left(c_{-i}, d_{i}\right)-U_{i}(c)\right) \leq 0, \forall i \in N, \forall c_{i} \in C_{i}, \forall d_{i} \in C_{i} .
\end{aligned}
$$

So the dual of this problem also has optimal value equal to zero. But this dual is

$$
\begin{aligned}
& \text { choose } \alpha \text { and } \beta \text { to minimize } \beta \\
& \text { subject to } \\
& \alpha_{i}\left(d_{i} \mid c_{i}\right) \geq 0, \quad \forall i \in N, \forall c_{i} \in C_{i}, \forall d_{i} \in C_{i}, \\
& \Sigma_{i \in N} \Sigma_{d_{i} \in C_{i}} \alpha_{i}\left(d_{i} \mid c_{i}\right)\left(U_{i}\left(c_{-i}, d_{i}\right)-U_{i}(c)\right)+\beta \geq 0, \quad \forall c \in C \text { such that } c_{j} \neq e_{j}, \\
& \Sigma_{i \in N} \Sigma_{d_{i} \in C_{i}} \alpha_{i}\left(d_{i} \mid e_{i}\right)\left(U_{i}\left(e, i, d_{i}\right)-U_{i}(e)\right)+\beta \geq\left(U_{j}(e)-U_{j}\left(e, j, b_{j}\right)\right), \quad \forall e_{-j} \in C_{-j} .
\end{aligned}
$$

So there exists some vector $\alpha$ that satisfies these constraints with $\beta=0$. But now consider a vector $\alpha$ that is the same as this $\alpha$ in every component except that $\hat{\alpha}_{j}\left(b_{j} \mid e_{j}\right)=\alpha_{j}\left(b_{j} \mid e_{j}\right)+1$. Then this vector satisfies the following three properties:

$$
\begin{aligned}
& \hat{\alpha}_{i}\left(d_{i} \mid c_{i}\right) \geq 0, \quad \forall i \in N, \forall c_{i} \in C_{i}, \forall d_{i} \in C_{i}, \\
& \hat{\alpha}_{j}\left(b_{j} \mid e_{j}\right)>0 \text {, and } \\
& \sum_{i \in N} \sum_{d_{i} \in C} \hat{\alpha}_{i}\left(d_{i} \mid c_{i}\right)\left(U_{i}\left(c_{-i}, d_{i}\right)-U_{i}(c)\right) \geq 0, \forall c \in C .
\end{aligned}
$$

Multiplying such a vector $\hat{\alpha}$ by a small positive scalar if necessary, we can also make it satisfy 


$$
\Sigma_{d_{i} \in C_{i}} \hat{\alpha}_{i}\left(d_{i} \mid c_{i}\right) \leq 1, \quad \forall i \in N, \quad \forall c_{i} \in C_{i} .
$$

Then increasing $\hat{\alpha}_{i}\left(c_{i} \mid c_{i}\right)$ (which has a zero coefficient in the third of the three properties listed above) as necessary, we can make a vector $\alpha$ that also satisfies

$$
\Sigma_{d_{i} \in C_{i}} \hat{\alpha}_{i}\left(d_{i} \mid c_{i}\right)=1, \quad \forall i \in N, \quad \forall c_{i} \in C_{i} .
$$

Then this $\hat{\alpha}$ is a dual vector such that $\hat{\alpha}_{j}\left(b_{j} \mid e_{j}\right)>0$, as required by Proposition 2.

Q.E.D. 


\section{REFERENCES}

Aumann, R. J., "Subjectivity and Correlation in Randomized Strategies, " Journal of Mathematical Economics 1:67-96 (1974).

Chvatal, V., Linear Programming, New York: W. H. Freeman (1983).

Dhillon, A, and J.-F. Mertens, "Perfect Correlated Equilibria," CORE working paper, Universite Catholique de Louvain (1992).

Damme, E. van., Stability and Perfection of Nash Equilibria, 2nd ed., Berlin: Springer-Verlag (1991).

Karlin, S., A First Course in Stochastic Processes, New York: Academic Press (1969).

Hart, S., and D. Schmeidler, "Existence of Correlated Equilibria," Mathematics of Operations Research 14:18-25 (1989).

Myerson, R. B., "Acceptable and Predominant Correlated Equilibria," International Journa1 of Game Theory 15:133-154 (1986).

Nash, J. F., "Noncooperative Games," Annals of Muthematics 54:289-295 (1951).

Nau, R. F., and K F. McCardle, "Coherent Behavior in Noncooperative Games," Journal of Economic Theory 50:424-444 (1990).

Selten, R., "Reexamination of the Perfectness Concept for Equilibrium Points in Extensive Games," International Journal of Game Theory 4:25-55 (1975). 